
allemande

45-2 | 2013

Images et discours de la nation

\title{
Gegenläufigkeit der Tell- und Alpen-Diskurse in der Schweizer bildenden Kunst des 20. Jahrhunderts
}

Integrativer Tell- und Alpen-Mythos, identitätsstiftende Tell- und AlpenEmblematik und ihre korrosiven Gegendiskurse

Barbara Lafond

\section{CpenEdition}

Journals

Édition électronique

URL : https://journals.openedition.org/allemagne/1545

DOI : $10.4000 /$ allemagne. 1545

ISSN : 2605-7913

Éditeur

Société d'études allemandes

Édition imprimée

Date de publication : 30 décembre 2013

Pagination : 403-416

ISSN : 0035-0974

Référence électronique

Barbara Lafond, "Gegenläufigkeit der Tell- und Alpen-Diskurse in der Schweizer bildenden Kunst des

20. Jahrhunderts", Revue d'Allemagne et des pays de langue allemande [Online], 45-2 | 2013, Online erschienen am: 29 Juli 2019, abgerufen am 19 Mai 2021. URL: http://journals.openedition.org/ allemagne/1545 ; DOI: https://doi.org/10.4000/allemagne.1545 


\title{
Gegenläufigkeit der Tell- und Alpen-Diskurse in der Schweizer bildenden Kunst des 20. Jahrhunderts
}

\author{
Integrativer Tell- und Alpen-Mythos, \\ identitätsstiftende Tell- und Alpen-Emblematik \\ und ihre korrosiven Gegendiskurse
}

- Barbara Lafond*

Die Fragestellung der Imagologie ist die nach den Bildern der Alterität auf der Ebene der Gesellschaft oder einer kulturellen Gemeinschaft. Die Imagologie lässt sich ebenso fruchtbar auf die Selbstporträtierung einer Gesellschaft anwenden; insofern ist sie auch für die Analyse des Selbstverständnisses einer Gesellschaft relevant. Das imagologische Zeichensystem folgt einer zeit- und kulturbedingten semantischen Grammatik; es ist kodiert und durch die Rezipienten mehr oder weniger dekodierbar.

$\mathrm{Zu}$ den prägnantesten nationalen Vorstellungsbildern der Schweiz zählen Wilhelm Tell und die Alpen. Schweizerische Identitätsbilder wie die Alpen sind vor allem von den Aufklärern erstellt worden, um einen gesamtschweizerischen Patriotismus zu fördern. Die Vorstellung des „Schweizeralpenlandes“(1), dessen Geschichte und Kultur hauptsächlich durch die Alpen bestimmt sind, wurde von Lavater geprägt. Der Landschaftsraum des Schweizeralpenlandes ist seither ein konstitutives Merkmal seiner Identität, und dies in weitaus größerem Maße als in der Alpenrepublik Österreich ${ }^{(2)}$. Diese Berufung auf eine

* Maître de conférences habilitée à diriger des recherches, Université de Strasbourg.

1 Den Begriff „Schweizeralpenland“ prägte der Zürcher Pfarrer, Schriftsteller und Philosoph Johann Caspar Lavater. Die Identifikation von Schweiz und Alpen wurde ab dem 18. Jahrhundert zu einem wichtigen Element nationaler Identitätsbildung. Vergleiche dazu die Untersuchung von Guy P. MARCHAL, „Das ,Schweizeralpenland': eine imagologische Bastelei“, in: Guy P. MARCHAL und Aram MATTIOLI (Hg.), Erfundene Schweiz. Konstruktionen nationaler Identität. La Suisse imaginée. Bricolage d'une identité nationale, Zürich, Chronos Verlag, 1992.

2 Günter Schweiger, Österreichs Image in der Welt. Ein Vergleich mit Deutschland und der Schweiz, Wien, Service-Fachverlag, 1992, S. 177: „Bilder von sommerlichen Landschaften werden, auch wenn sie aus Österreich stammen, vorrangig mit der Schweiz in Verbindung gebracht. Selbst den Großglockner, diese nationale Ikone Österreichs, ordnen sogar Schweizer öfter ihrem eigenen Land als Österreich zu." 
unverwechselbare Landestopographie ist auch in anderen multikulturellen Gesellschaften, in denen eine einheitliche "Sprache“ als verbindendes Element fehlt, eine bevorzugte Diskurspraktik. Die Alpen-Bilder dienten in den Krisenzeiten nach 1848 immer wieder zur Definition und Festigung nationaler Identität, fungierten in der Malerei in den heroischen pathos-geladenen Gebirgsszenen des 19. Jahrhunderts als nationales Motiv ${ }^{(3)}$.

\section{„homo alpinus helveticus“}

Die Wesensbestimmung des Schweizers aus der alpinen Situation heraus lag in der überkommenen Tradition der Identitätsrepräsentation vor. Bereits der Waadtländer Sirice Bridel (1757-1845) hatte den „wahren Schweizer“ charakterisiert: „Der Hirte des Oberwallis ist etwas, er ist Schweizer. Immer derselbe auf dem Gipfel des Gotthards, wo er seine Herde weidet. Seine viel zitierte Formel lautet: ,Ex alpibus salus patriae! (,Aus den Alpen das Heil des Vaterlandes!')“(4). Bereits Johann Jakob Scheuchzer (1672-1733) hatte das Geschichtsbild von dem „Alten Eidgenossen“ mit dem „homo alpinus“ gleichgestellt. Die Wesensmerkmale der vorbildhaften Vorfahren waren geformt durch Klima und alpine Umwelt; auch der Bauernstand erhielt eine alpine Konnotation. Urs Balthasar, der verehrte Gründervater der helvetischen Gesellschaft, schrieb 1762 in seinen letzten Wünschen: „Der Charakter dieser Nation stehe mit ihrer rauhen, bergichten und ungehobelten Landschaft in vollkommenem Verhältnis." Von da an bilden die Alpen einen konstitutiven Bestandteil der schweizerischen Wesens$\operatorname{art}^{(5)}$. Doch diese Vorstellungen von einer alpinen Wesensart führen in die gefährliche Nähe von Rassentheorien Ende des 19. und zu Beginn des 20. Jahrhunderts ${ }^{(6)}$.

\section{Wilhelm Tell - Konstruktion und Dekonstruktion}

Bei der nationalen Ikone des Wilhelm Tell handelt sich um ein Zeichensystem, in welchem mittelalterliche Geschichte und Alpen bestimmende Hauptbedeutungsfelder sind, eine in der bildenden Kunst ergiebige Semantik. Tell ist ein Beispiel für die Beziehung von Kunst und Politik, wobei die irrationalen Traditionsbezüge der Bilder den intuitiven Zugang zum modernen Staat erleichtern.

Für Jean Starobinski braucht die Nation „symbolische und mythische Selbstdarstellung“(7). Die Geschichte als besonders wichtiges Integrationsmittel lässt sich in der Abbreviatur des Bildes für Tausende in einem Blick in Erinnerung rufen. Das zeigt die Geschichte der Wilhelm Tell-Denkmäler. Gerade auf dem Feld der bildenden Kunst lassen sich erste Ansätze zu einer staatlichen Kulturpolitik bilden. So begann der bürgerliche Staat, sich gegen Ende des 19. Jahrhunderts selbst darzustellen.

3 Barbara Lafond-Kettlitz, „Die Alpen in Literatur und Malerei. Albrecht von Haller, Caspar Wolf, Ludwig Hohl, Ferdinand Hodler", Études Germaniques-Esthétique-Histoire des arts, Études germaniques, 4 (2009), Paris, Klincksieck, S. 911-933.

4 Guy. P. Marchal, „Die ,Alten Eidgenossen“ im Wandel der Zeiten“, in: Innerschweiz und frühe Eidgenossenschaft. Jubiläumsschrift 700 Jahre Eidgenossenschaft, Bd. 2, Olten, 1990, S. 307-403, hier S. 345.

5 S. dazu auch den Romanisten Ernest Bovet, „Réflexions d'un Homo Alpinus“, Wissen und Leben, 3 (1908), S. 296-299.

6 Georg KREIS, „Der ,homo alinus helveticus‘. Zum schweizerischen Rassendiskurs der 30er Jahre“, in: Marchal/Mattioli (Hg.), Erfundene Schweiz (Anm. 1), S. 175-190.

7 Jean Starobinski, 1789. Les emblèmes de la raison, Paris, Flammarion, 1979. 
Der Anstoß zum ersten großen Tellbild der Zeit, zur Neuausmalung der Tellskapelle am Vierwaldstättersee kam 1865 zwar von König Ludwig II. von Bayern, doch die Urner wandten sich lieber an den schweizerischen Kunstverein; der Historienmaler Ernst Stückelberg gewann den Wettbewerb, denn er schuf 1880-82 eine getreue Umsetzung von Schillers Schauspiel in vier Szenen (Tells Sprung - der Rütlischwur - der Apfelschuss - Gesslers Tod).

1891 sollte ein Denkmal in Altdorf errichtet werden: „Wilhelm Tell ist als freiheitstolzer, kühner, entschlossener Mann, in der landesüblichen Bauerntracht seiner Zeit darzustellen.“ Preisträger wurde der Bildhauer Richard Kissling mit seinem klassizistisch-heroischen Stil, denn er stellte Tell mit dem Knaben dar: „Kisslings Tell setzt den Gestus des Schiller-Wortes am besten plastisch um, indem sich der Junge eng an den Vater anschließt und dieser ihn mit dem linken Arm festhält, während der rechte die Armbrust schultert: Schutz und Trutz" ${ }^{(8)}$. Tells Heroisierung erfolgt über das kleine Kind an seiner Seite, das, die Blickrichtung nach oben, Tells Größe noch besser hervorhebt; es hat sozusagen eine konterkarierende Funktion; die Vater-SohnStatue als ein gestisches Kürzel für das Vaterland.

1897 löste Ferdinand Hodler die Tell-Figur aus dem Kontext der Darstellung von Gesslers Tod und setzte sie mitten ins Bild.

„Die Natur steht ganz im Dienst der Inszenierung von Tells Auftritt, er scheint aus einem Riss in den Wolken zu treten, welcher mit einer blauen Parallelkomposition Tiefe ins Bild bringt. Die Armbrust wird genau parallel zur Hochachse des Bildes gerichtet zu einem dem Betrachter entgegen gestreckten Symbol. Die Geste der erhobenen Hand wird durch den zum Schalltrichter erweiterten Mund verschieden deutbar. Indem sich die imaginäre leere Sprechblase von Hodlers Tell fast beliebig auffüllen lässt, wird Tell so zum Träger jener allgemeinen Werte, über welche sich die Gesellschaft integriert. Nicht einfach im dargestellten plakativen Freiheitspathos, sondern in seiner verallgemeinernden Interpretierbarkeit liegt dementsprechend der Grund, dass der Tell zum bekanntesten Gemälde Hodlers wurde und mithalf, seinen flächigen symbolistischen Jugendstil gegen den herrschenden Historismus durchzusetzen. Hodlers Tell wird zum patriotischen Versatzstück, das eine eigene Wirkungsgeschichte entwickelt, zur nationalen Kunst“(9).

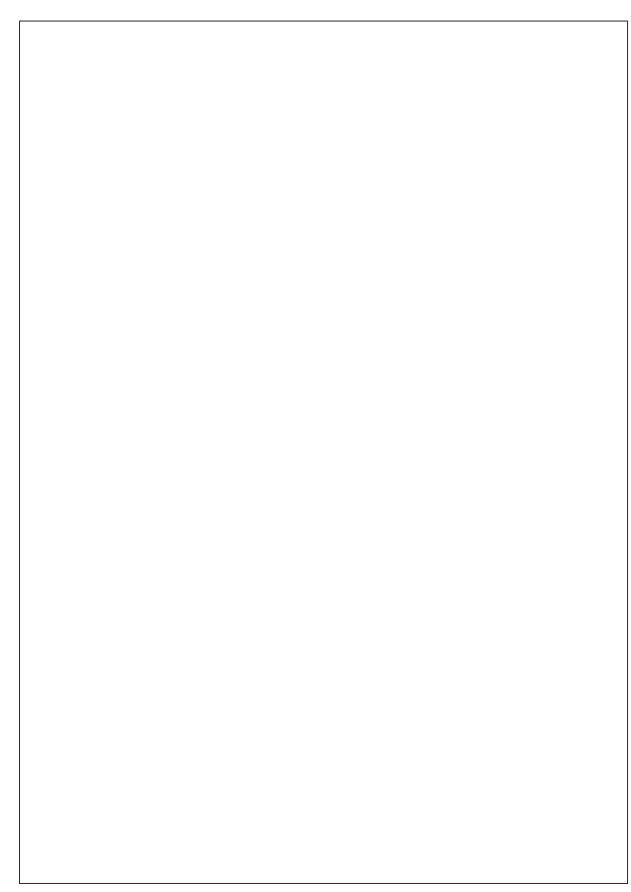

Wilhelm Tell, Gemälde Ferdinand Hodler (1897), in: Lilly Stunzi, Jean Rodolphe de Salis, Tell. Werden und Wandern eines Mythos, Bern/Stuttgart, Hallwag Verlag, 1973, S. 307.

8 Peter UTz, Die ausgehöhlte Gasse. Stationen der Wirkungsgeschichte von Schillers „Wilhelm Tell“, Königstein, Forum Academicum in der Verlagsgruppe Athenäum, Hain, Hanstein, 1994, S. 180.

9 Ebd., S. 184-185. 
Hodlers Tell erinnert an die Figur des Moses, der mit den Gesetzestafeln vom Berg Sinai herabsteigt. Doch seine Armbrust wurde zum Gütezeichen, als Qualitätssiegel von der Schweizer Industrie übernommen, was von der ökonomischen Instrumentalisierung des Tell-Mythos zeugt. Für Irritation sorgten auch René Magrittes parodistische Tell-Darstellung und Salvador Dalis La vieillesse de Guillaume Tell (1931). Der mit patriotischen Erwartungen aufgeladene Blick des Betrachters prallt nämlich an einer Leinwand ab. Tell hält sich versteckt, wenn überhaupt, ein merkwürdiger Schatten, seine Abwesenheit scheint das Ende des Tellkultes anzukündigen.

In den achtziger Jahren ist die Dekonstruktion bereits ein Fakt: Tell sei „eine Art heroischer Kleiderbügel, an dem sich die verschiedensten parteipolitischen und wirtschaftlichen Interessemäntelchen je nach Bedarf aufhängen lassen “ ${ }^{(10)}$. Dies äußert sich auch in Friedrich Dürrenmatts Buch für Schweizer Kinder: Die Heimat im Plakat. Dürrenmatt zeichnet eine Serie von Karikaturen, die das Idol parodieren. Der Schütze kann sein Ziel verfehlen, ein Pfeil trifft die Stirn des Jungen. Die Inschrift: „Tell trank Kläfner“ dient als Erklärung dazu: „Kläfner ist ein Wein, den Dürrenmatt verabscheute ${ }^{(11)}$. So zeigen auch kitschige lebensgroße Wilhelm Tell-Bären aus Plastik die Trivialisierung und Vermarktung des Mythos. Nicht nur Dekonstruktion, sondern Transgression nimmt Adam Tellmeister in seiner künstlerischen Auseinandersetzung mit dem TellMythos vor. Er will den Tell neu besetzen und versucht, in Tells Spannungsverhältnis von Tyrannenmörder und heutigem Terroristen eine Gegenwartsfigur herauszubilden und in Umlauf zu bringen, als neuen Schweizer ${ }^{(12)}$. So fertigte er eine Installation mit großformatigen Zeichnungen und verschiedenen Totenmasken von Tell, die neben denen von Gudrun Ensslin und Ulrike Meinhof stehen. Er pervertiert die mythische Figur, wenn seine Zeichnungen unter dem Motto „böser Tell“ in der Ausstellung eines Berliner Kindergartens auf die Heldensaga von Tell als Akt des Kindesmissbrauchs verweisen - als Signum postmoderner Dekonstruktion des Nationalmythos.

\section{Die Alpen als eidgenössisches Integrationssymbol}

In Philosophie und Ästhetik geht die Wahrnehmung der Bergwelt als Harmonie zurück auf die Umwertung der Alpen um ungefähr 1700 von den schrecklichen Alpen, dem locus horribilis, zu den erhabenen Alpen; sie wurden zum Inbegriff des Schönen, eine Änderung, die mit der „Theodizee“ von Gottfried Wilhelm Leibniz zusammenfiel ${ }^{(13)}$. Sie waren fortan Ausdruck einer Schöpfungsordnung, wurden zum Ort des Erhabenen, ganz im Sinne von Immanuel Kant ${ }^{(14)}$ und Friedrich Schiller, welche die

10 Uli Windisch, Florence Cornu, Tell im Alltag, Zürich, Edition M., 1988, S. 343-350.

11 Friedrich Dürrenmatt, Die Heimat im Plakat. Ein Buch für Schweizer Kinder, Zürich, Diogenes, 1963, S. 23.

12 Adam Tellmeister, geboren 1961 in Sumiswald Bern, eigentlich Adam Meister, ist ein Schweizer Künstler. Er lebte und arbeitete über 20 Jahre lang als „sans-papiers“ in Deutschland, seit 1989 in Berlin, er versteht sich als Kunstfigur Tellmeister.

13 Petra Raymond, Von der Landschaft im Kopf zur Landschaft als Sprache. Die Romantisierung der Alpen in den Reiseschilderungen und die Literarisierung des Gebirges in der Erzählprosa der Goethezeit, Tübingen, Niemeyer (Studien zur deutschen Literatur, 123), 1993, S. 11-165, S. 185-230.

14 Immanuel KANT, „Beobachtungen über das Gefühl des Schönen und Erhabenen“, in: Kants populäre Schriften, Berlin, Reimer, 1911, S. 60-80 und 111-125. Romantische Kritik der Ansichten von Kant 
„Erhabenheit der natürlichen Landschaft“als Zustand der Freiheit definieren. Die Alpen wurden so Verkörperung subjektiver Freiheit. Göttliche Prädikate wurden mit denen des Raumes identifiziert, wodurch eine ästhetische Kategorie entstand, die Ästhetik des Naturerhabenen, die die Vermittlung des Begriffs der Unendlichkeit erbrachte.

Der Alpen-Mythos war ein beliebtes Motiv in der Literatur. Am 22. Juli 1803 hatte Goethe durch seine Erzählungen die Vierwaldstätter-Szenerie für den Tell vor dem inneren Auge Schillers entstehen lassen. Und er skizzierte auf seiner ersten Schweizer Reise im Sommer 1775 die Alpen als Mythen, wie Roland Barthes sie definieren sollte: „Le mythe, c'est-à-dire une ,parole dépolitisée ‘ qui évacue la qualité historique des choses, les purifie, les innocente, les fonde en nature et en éternité “(15). Georg Simmel sprach in seinem Aufsatz Die Alpen von der transzendentalen Qualität der Alpen, die sie „durch die ungefüge Masse des Gestaltlosen erst entfalten“ “(16).

Doch die Alpen waren auch Kultgegenstand der helvetischen Politik, denn sie waren für das sprachlich, konfessionell und kulturell vielfältig segmentierte Land ein geeignetes Integrationsmittel. Sie galten als „schützendes, unerschütterliches Zentrum in einer sich immer schneller ändernden Welt“ (17), „als Schutzwälle und damit als Sinnbild der im Inneren des Landes gewahrten Freiheit“ ${ }^{\text {(18) }}$. Im politischen Diskurs wurden die Alpen idealisiert und sakralisiert. Die helvetischen Politiker bemühten sich in ihren Reden und in ihrer Kulturpolitik um einen demonstrativen Alpen-Bezug, weil sie glaubten, dass die Landschaft günstige Wirkungen auf den Zusammenhalt und ihre Institutionen ausübe. Dies hinterließ auch deutliche Spuren in der Alpenmalerei.

Die Entstehung der Schweiz wäre ohne Brücken und Alpenübergänge undenkbar: Sie sind ein typisches Motiv in der Alpenmalerei. So die Teufelsbrücke von Caspar Wolf (1735-1783), die er vor allem mit Lichtkontrasten gestaltete ${ }^{(19)}$. Er gilt neben Pierre-Louis de la Rive (1753-1817) als Entdecker des Hochgebirges für die Schweizer Landschaftsmalerei, denn er befreite die Gebirgslandschaft von ihrer vormaligen Funktion, imposanten Hintergrund für Staffageszenen zu bilden. Sie wird eigenständiger Bildgegenstand und somit autonomes Subjekt in der Kunst der Zeit der Vorromantik. Caspar Wolf erkundete mit aufklärerischen Naturwissenschaftlern die Bergwelt, wurde zum gelehrten Maler, der genau kennt, was er abbildet. Seine Malerei war weitgehend verschollen und ist erst seit 1948 in Schweizer Museen vertreten. Er

und Burke über das Erhabene: A. W. SCHLEgel, Vorlesungen über schöne Literatur und Kunst, hg. von Jakob Minor, Heilbronn, Gebr. Henninger, 1884, I, S. 58-112.

15 Roland Barthes, Mythologies, Paris, 1957, S. 216. „Der Mythos, entpolitisierte Worte, die die historische Dimension der Dinge ausblendet, sie reinigt, sie in den Zustand der Unschuld versetzt und sie mit Natur und Ewigkeit verschmelzt.“

16 Georg Simmel, Zur Ästhetik der Alpen (1911), in: Ders., Aufsätze und Abhandlungen 1909-1918, Bd. 1 (= Gesamtausgabe, Bd. 12), hg. von Rüdiger Kramme, Angela Rammstedt, Frankfurt am Main, 2001, S. 162-169, hier S. 165.

17 Hans Peter Treichler, Abenteuer Schweiz. Geschichte in Jahrhundertschritten, Zürich, MigrosGenossenschafts-Bund, 1991, S. 203.

18 François de Capitani, „Die Alpen: Wiege der Freiheit“, in: Dario Camboni, Georg Germann (Hg.), Zeichen der Freiheit. Das Bild der Republik in der Kunst des 16.-20. Jahrhunderts. Ausstellungskatalog Nr. 3. Bern, 1991, S. 393-454, hier S. 395.

19 Caspar Wolf, Die Teufelsbrücke bei Schöllenen, 1777, Öl auf Leinwand, 82 x 54 cm, Aarau, Aargauer Kunsthaus, Depositum der Gottfried Keller-Stiftung. 
übte einen starken Einfluss auf die folgenden Generationen aus, und selbst zeitgenössische Künstler haben sich mit seiner Kunst im speziellen auseinandergesetzt, dies gilt für Hugo Suter, Michael Biberstein, Gloria Friedmann, Per Kirkeby, Richard Long und Anna Winteler ${ }^{(20)}$.

Da die Alpendarstellungen als kulturgeschichtliches Phänomen es auch ermöglichen, Bezüge zu nationalen Identitätsstrukturen und zum kulturellen Gedächtnis herzustellen, so fungieren sie in den heroischen Gebirgsszenen des 19. Jahrhunderts als nationales Motiv. Die Hinwendung der Kunst des 19. Jahrhunderts zu realistischen Darstellungen historisch-nationaler Themen ist ein gesamteuropäisches Phänomen, das auch für die Schweiz zutrifft. Die Alpenthematik wurde zur Verkörperung der Schweizer Kunst, zur nationalen Ikone, zum Mythos der Schweiz ${ }^{(21)}$. Die politische Konsolidierung nach der Bundesverfassung von 1848 begünstigte die bewusste Schaffung einer bis dahin nicht bestehenden schweizerisch-nationalen Malschule. Die Verwirklichung der patriotischen Aspiration geschah zuerst durch die Genfer Maler Alexander Calame ${ }^{(22)}$ und François Diday ${ }^{(23)}$. Letzterer war mit seiner heroischen Vision der einflussreichste und populärste. In dramatischen, pathosgeladenen Landschaften, die auch nationalgeschichtliche Begebenheiten darstellen, wurde die Erhabenheit der Berge zum ersten Mal mit den zerstörerischen Naturelementen verbunden. Die Ausdrucksmöglichkeiten weiteten sich auf Wolken und Sturmszenen aus. Gustave Doré illustrierte dramatische Szenen wie den Absturz der Whympergruppe am Matterhorn. Die Natur ist als künstlerischer Ausdruck der Nation interpretiert worden. Gottfried Kellers Grüner Heinrich gesteht freimütig ein: „Mit der Gedankenlosigkeit der Jugend und des kindischen Alters hielt ich die Schönheit des Landes für ein historisch-politisches Verdienst, gewissermaßen für eine patriotische Tat des Volkes und gleichbedeutend mit der Freiheit selbst“(24). Der Blick auf die Landschaft erfuhr eine politisch gefärbte Perspektive.

$\mathrm{Zu}$ den Bildern des kollektiven Imaginären gehört auch der Gotthard, er wird zum Inbegriff des schweizerischen Staates: Gotthard-Staat ${ }^{(25)}$. Die Gotthardpost, ein 1873 von Rudolf Koller geschaffenes Gemälde ist ein wichtiges Beispiel dafür. Im Auftrag der Direktion der Schweizerischen Nordostbahn malte er den Gotthard: „Zum einen ist es der Titel, der bei historisch vorgebildeten Betrachtern ein Wissens- und Assoziationsspektrum anklingen lässt. Der Gotthard als Beweis für die geglückte und sichere Alpenüberquerung, für die bewundernswerte Ingenieurleistung eines Eidgenossen, und vor allem der Gotthard als Symbol für die Erhabenheit der Umwelt - dies

20 Stephan Kunz, Beat Wismer, Wolfgang Denk (Hg.), Die Schwerkraft der Berge 1774-1997. Katalog anlässlich der Ausstellung im Aargauer Kunsthaus Aarau und der Kunsthalle Krems 15. Juni bis 24. August 1997; 7. September bis 23. November 1997, Trans alpin: 1, Basel, Stroemfeld/Roter Stern, 1997, S. 12.

21 Siehe dazu: Peter Utz, „Alpen auf dem Papier. Literarische Erosionsformen des Alpenmassivs bei Robert Walser“, in: Marchal/Mattioli (Hg.), Erfundene Schweiz (Anm. 1), S. 313-326. Alexandre Calame (1810 Arabie, Gemeinde Corsier-sur-Vevey - 1864 Menton). François Diday (1802 Genf - 1877 Genf). Gottfried Keller, Minister Stapfer und die Künstlergesellschaft in Bern, Thun, 1954, S. 9-12, hier S. 11. Bei P. Utz, Die ausgehöhlte Gasse (Anm. 8), S. 122. 
verbindet sich zu einer Allegorie auf die Eigenschaften der Nation und ermöglicht einen Begriff von Heimat, der über das Landschaftliche hinaus in das soziale und politische Selbstverständnis reicht“(26).

Das Panorama war die Sehweise, die die Gesellschaft des 19. Jahrhunderts prägte, die ideale Sichtweise der politischen Kultur der Eidgenossenschaft als föderalistische Rundschau, die Berge bildeten den dramatisch gestalteten Schwerpunkt. So zeigt das Bourbaki-Panorama (1881) von Edouard Castres den Übertritt der im Januar 1871 vor den Deutschen fliehenden geschlagenen Truppen. Dieses Panorama hat viel zum Mythos der Schweiz als Asylland beigetragen ${ }^{(27)}$. Zu dem Team der Maler gehörte auch Ferdinand Hodler, der ebenso wie Edouard Castres im Panorama porträtiert ist: Castres als Rotkreuzhelfer, Hodler als Berner Soldat. „Die Schweizer Historienmalerei verfolgte nationale und soziale Integration, wirkte nach innen, indem sie auf Vergangenheit verpflichtete “(28). Eine der am weitesten verbreiteten Reproduktionen und Höhepunkt der eidgenössischen Selbstfindung waren Ferdinand Hodlers Berge: „Das Hodlergebirge verleiht der schweizerischen Landschaft eine Dominante von überragender Wucht und Stärke. Es hebt die Schweiz als relative Einheit mehr oder weniger ab von den umgebenden Nachbarländern “ ${ }^{(29)}$. Eine patriotische Ästhetik charakterisiert auch die Kunstpolitik der Zwischenkriegszeit, in dieser Ambiance hatten die Modernen, die abstrakte, surrealistische ungegenständliche Kunst einen schweren Stand ${ }^{(30)}$.

\section{Gegenläufige Alpendiskurse in der Malerei}

Die ideologische Überhöhung der Alpen in der Schweiz wird um die Jahrhundertwende im politischen, literarischen und kulturellen Diskurs unmerklich von gegenläufigen Alpen-Bildern unterhöhlt. „Während eine ganze Dichtergeneration daran arbeitet, das alpine Urgestein mit der Knetmasse des Alpenromans zu überhöhen, wirken andere Autoren an der literarischen Erosion dieses Massivs“ “(31). Dies gilt auch für den Bereich der bildenden Kunst und lässt sich am Beispiel des Malers Karl Walser, des Bruders von Robert Walser, nachvollziehen. Er erodiert die heroische Alpenmalerei durch Verkleinerung. So sein Bild Aussicht auf die Alpen (1899), das sein Bruder im Prosastück Leben eines Malers kommentiert. „Mitten im prächtigen Bilde liegt unter Tannen wieder einmal leider Gottes, wie es scheint, so ein Strick und Tagedieb von träumendem, faulenzendem Monsieur Faulpelz. Reizend ist angedeutet, wie die Natur in ihrer Ruhe ihr schönstes Schöne darbietet, was alles vom jungen Menschen auf dem

26 www.gymmuenchenstein.ch/stalder/klassen/sa/kunsthtm/koller.htm

27 Das Bourbaki-Panorama Luzern zeugt als eines der ganz wenigen noch erhaltenen Riesenrundgemälde weltweit in einzigartiger Weise von der Mediengeschichte des 19. Jahrhunderts. Es ist 112 Meter lang und 10 Meter hoch und zeigt die französische Armée de l'Est des Generals Charles Denis Sauter Bourbaki bei ihrem Übertritt in die Schweiz am Ende des Deutsch-Französischen Krieges von $1870 / 71$.

28 Georg Kreis, Mythos Rütli. Geschichte eines Erinnerungsortes, Zürich, Orell Füssli, 2004.

29 Hermann GANZ, „Zur Entstehung der nationalen Schule in der schweizerischen Kunst“, Die Schweiz, 25 (1921), S. 38.

30 Hodlers Stil entsprach dieser patriotischen Ästhetik.

31 P. Utz, „Alpen auf dem Papier“ (Anm. 21), S. 313-327. 
Grasboden weiter durchaus nicht beachtet wird. Muss das ein träger Kerl sein! Ist es etwa ein Dichter? Hoffentlich doch wohl nicht“"(32).

Die Alpenkette ist weit entrückt mit heroischen Anklängen; statt der in der Alpenliteratur und -malerei der Zeit obligatorischen Vertikalität ist hier die Horizontalität betont. Im Blickfang befindet sich eine träumende Taugenichtsfigur, das Gegenteil zum homo alpinus aus dem Bergroman. Das Bild kann auch als eine Reflexion über Alpenmalerei und Alpendiskurs gedeutet werden: Der Dichter hat sein eigenes Gebiet, ist von den kollektiven Alpenträumen getrennt. Im Gewand der Ironie äußert Robert Walser seine Aversion gegen die patriotisch motivierte Alpenmalerei: „Mir fiel heut früh ein, mir zu sagen, niemand, wer er sei, dürfe sich herausnehmen, den Staat an die Wand zu malen, ein Gebilde seines Vaterlandes zu zeichnen, seine Heimat zu lobpreisen, weil hinter solchem unpassenden, unangemessenen Lob der Teufel lauere“ ${ }^{\text {(33). }}$.

Obwohl heldischer Alpinismus und Alpenmythos in den Dreißiger Jahren im Namen der Landesverteidigung stark heroisiert und in Kunst und Film mit dem Bezug Natur-Geschichte operiert wurde, gab es Gegenentwürfe. Der Schweizer Schriftsteller Ludwig Hohl zerstörte den Mythos der Alpen als Ort der subjektiven und der helvetischen Freiheit. Er gestaltete die Alpenlandschaft in einem Aquarell $^{(34)}$, das mit seinen geometrischen Formen und symmetrischen Farbkontrasten an Hodlers „Parallelismus“ erinnert. Doch seine Berglandschaft ist belebt; in der Mitte im Vordergrund des Bildes steht ein Murmeltier einsam in aufrechter Haltung auf einem großen schwarzen Felsblock: es gibt nur Absturzgefahr. In Untersicht sind in weiß-blauen Tönen pyramidenartige Gletschergipfel zu sehen, die sich in die Ferne verlieren. Unter dem Bild steht ein Vers aus Goethes Faust: „Hast du Begriff von Oed' und Einsamkeit?“ “(35). Dieser explizite Bezug zu einem literarischen Werk dient der Erschließung des Bildes und zeigt die enge Beziehung zwischen der Semantik der Landschaftmalerei und dem literarischen Werk. Hohls Alpen sind Chiffre für das Gefühl des „Aus-der-Welt-Gestoßenseins“, der Heimat- und metaphysischen Obdachlosigkeit. In seiner Novelle Bergfahrt nimmt er explizit Bezug auf Piranesis carceri $^{(36)}$.

Der politische Symbolgehalt der Alpen für die Schweiz erfuhr in den siebziger Jahren schließlich eine fortschreitende Umwertung, sie sind nicht mehr Signum der Freiheit,

32 Robert WALSER, Das Gesamtwerk, hg. von Jochen Greven, Genf/Hamburg, Kossodo, 1966-1975, III, S. 149.

33 Robert Walser, Europas schneeige Pelz-Boa. Texte zur Schweiz, hg. von Bernhard Echte, Frankfurt am Main, Suhrkamp Verlag, 2003, S. 100.

34 Ludwig Hoнl, Hast Du Begriff von Oed' und Einsamkeit?, Aquarell, in Privatbesitz Werner Morlang, Zürich.

35 Goethe, Faust II, Vers 6227.

36 Ulrike Gauss, „Giovanni Battista Piranesi - Die poetische Wahrheit. Die Radierungen“, in: Ausstellungskatalog Graphische Sammlung der Staatsgalerie Stuttgart, Stuttgart, 1999. „Piranesi Giovanni Battista, ital. Kupferstecher, Archäologe, Baumeister. 1720 bei Modena geboren, 1778 in Rom gest. Reihe düsterer Traumphantasien, (Carceri). [...] Die Carceri Piranesis von 1745 mit gespenstisch sich auftürmenden, verschachtelten, unheimlich leeren und bedrohlichen Bauten, Kerkervisionen gleich, sind Ausdruck seiner ausgesprochen malerisch-poetischen Phantasie." 
nicht mehr Schutzwall, sondern Gefängnis ${ }^{(37)}$. Das Gefängnis-Motiv ist übrigens in der schweizerischen Literatur rekurrent, um nur Paul Nizons Essay Diskurs in der Enge ${ }^{(38)}$, und Dürrenmatts Rede Die Schweiz als Gefängnis (1989) zu nennen.

\section{„Weg mit den Alpen! Freie Sicht aufs Mittelmeer!“}

Der Tod von Dürrenmatt (1990) und Frisch (1991) „markiert einen Einschnitt in der Deutschschweizer Literatur“ (39). Eine analoge Entwicklung ist in der bildenden Kunst festzustellen. „Eine neue Generation meldet sich zu Wort, eine Generation, der es nicht mehr um die Auseinandersetzung mit der Schweiz, um die Heimat als politische Kategorie, sondern höchstens noch als epische oder dramatische Kulisse für die Entwicklung der Figuren oder ökologische Probleme geht. Landesgrenzen spielen keine Rolle mehr“(40).

Bereits in den Jahren 1968-1970 waren die Alpen mögliches Untersuchungsfeld für die Kunst geworden, was eine Multiplizierung der Diskurse bedingte. Künstler näherten sich dem Motiv mit der Vielfalt der zeitgenössischen bildnerischen Medien und unter verschiedensten Aspekten: farbkräftiger Plakatstil, provozierende kräftige Farbgebung im Popstil, Hyperrealismus, Konzeptkunst. Der Versuch wurde gemacht, das nationale und kommerzielle Bild, das den Alpen anhaftet, zu durchbrechen; der gemeinsame Nenner vieler Künstler ist die Auflehnung gegen die politische und kommerzielle Überforderung der Berge in den Medien. „Eine neue Offenheit ist gefragt, helvetisch in ihrer Grundprägung und doch die Landesgrenzen sprengend, fernab von jedem chauvinistischen Insel-Kult “(41).

Am 31. Mai 1980 begannen in Zürich die Barrikadenkämpfe, die ,Opernhauskrawalle`, die rasch auf andere Städte übergriffen. Tausende, vorwiegend Jugendliche, demonstrierten unter dem drastischdadaistisch anmutenden Slogan „Nieder mit den Alpen! Freie Sicht aufs Mittelmeer!“ gegen einen städtischen Kredit von sechzig Millionen Franken für das Opernhaus in Zürich. Hingegen hatte die Jugendkultur zu dieser Zeit weder Begegnungsorte noch finanzielle Unterstützung. Die Autonome Jugendbewegung rüttelte damit am vermeintlich wichtigsten Identifikationssymbol der Schweiz: den Alpen. Dieser Satz kann auch als damaliger Versuch gewertet werden, „sich aus einer als zu stark empfundenen politischen Enge, sprich dem Sonderfallbewusstsein, zu befreien“(42).

37 Ruth Huber, „Dimension und Bild der Alpen in der Schweiz“, Die Alpen, Monatsbulletin des Schweizer Alpen-Clubs, 69 (1993), Nr. 9, S. 394-397.

38 Paul Nizon, Diskurs in der Enge, Verweigerter Steckbrief, hg. von Peter Henning, Frankfurt am Main, Suhrkamp, 1990, S. 175.

39 Hans Wysling, Streifzüge, Literatur aus der deutschen Schweiz, 1945-1991, Zürich, Schultheiss Polygraphischer Verlag, 1996, S. 251.

40 Mario Andreotti, „Aspekte und Tendenzen der neueren und neuesten Schweizer Literatur“, Literatur-Essay, Glarean Magazin, Dezember 2009.

41 Hermann Burger, „Schweizer Literatur nach 1968“, in: Ders., Als Autor auf der Stör, Frankfurt am Main, Fischer, 1987, S. 242.

42 Regula Zwahlen, Die Alpenfaszination in der Helvetik, Proseminararbeit, Univ. Luzern, 2003, S. 14. 
Im August 1998 fand im Kunsthaus Zürich die Ausstellung In den Alpen unter dem Titel Freie Sicht aufs Mittelmeer: junge Schweizer Kunst mit Gästen statt ${ }^{(43)}$. Die Kuratorin Bice Curiger hatte den ersten Teil des Slogans der Autonomen Jugendbewegung verboten mit der Begründung, der Horizont der lokalen Kunstszene habe sich seit den achtziger Jahren erheblich erweitert ${ }^{(44)}$. Das ist richtig, insofern die Alpenmalerei nicht mehr im Dienst von parteipolitischen oder nationalen Interessen steht, sondern ihre eigenen Wege geht.

Es ist relevant, dass dabei der deutsche Maler Gerhard Richter ${ }^{(45)}$ eine Schlüsselfigur darstellt mit seinem großen Fünfteiler Schweizer Alpen von 1969, mit den Abmalungen von Fotografien, die er vom Flugzeug aus in Vogelperspektive machte. „So wie ein einziges Schulterzucken ein großes Palaver beenden kann, reiht Richter die Alpen ein ins Repertoire verfügbarer Vorlagen. Er planiert den Mythos und stellt die Form aus, eine strukturalistische Tätigkeit“ (46). Er benutzte erstmals Fotografien als Vorlagen für Gemälde, ein Verfahren, das er danach regelmäßig aufgriff. Es handelt sich um eigene Aufnahmen, die er abmalend vergrößert und in Grau-Weiß auf die Leinwand überträgt und damit überhöht. Es geht ihm dabei um die Auseinandersetzung mit der Tradition. 1986 formulierte er sein Verhältnis zur Kunstgeschichte: „Und ich sehe mich als Erben einer ungeheuren, großen, reichen Kultur der Malerei, der Kunst überhaupt, die wir verloren haben, die uns aber verpflichtet“ ${ }^{\text {(47) }}$. Seine Alpen-Bilder stehen im intertextuellen Feld anderer Bilder der Kunstgeschichte. Er praktiziert den Rückgriff auf die Fotografie, die medial vermittelte Wirklichkeit, und destruiert somit den Mythos von der schöpferischen Unmittelbarkeit.

Die Schweizer Künstler Monica Studer (1960) und Christoph van den Berg (1962) führen einen neuen künstlerischen Umgang mit den Alpen. In ihrem Projekt Hôtel vues des Alpes, Bergtour 2008 kreieren sie rein virtuelle Landschaften und ein imaginäres Alpenhotel, in dem man sich virtuell ein Zimmer buchen kann. Mit der Computerkunst schreiben sie den Mythos der Alpen als Erholungsraum weiter, wobei jedoch jede nationale Konnotation fehlt.

Interessant ist ihre Reflexion über den Umgang mit klassischem Bilderbe aus einer transnationalen Perspektive, so ihr Bild Loch (2009) ${ }^{(48)}$, das in einem evidenten Bezug zu Caspar David Friedrichs Der Wanderer über dem Nebelmeer (1818) steht. Friedrichs Wanderer blickt als kleines Figürchen ganz versunken auf die erhabene Natur zu seinen Füßen. Sein Bild evoziert den Eindruck, dass die Natur zum Gegenstand religiöser

43 Freie Sicht aufs Mittelmeer: junge Schweizer Kunst mit Gästen, 5. Juni-30. August 1998, Kunsthaus Zürich, 6. Oktober-22. November 1998, Schirn Kunsthalle Frankfurt, Ausstellung und Katalog: Bice Curiger. - Zürich, Scalo, cop. 1998.

44 S. Anm. 43, Vorwort: „L'horizon considérablement élargi depuis les années quatre-vingt de la scène artistique locale."

45 Gerhard Richter (1932 Dresden), Maler, Bildhauer und Fotograf; von 1971 bis 1993 Professor für Malerei an der Kunstakademie Düsseldorf.

46 „Mythos pur und planiert“, Die Zeit, Feuilleton, 9.1.1998.

47 Hans-Ulrich Оввіsт (Hg.), Gerhard Richter: Text. Schriften und Interviews, Frankfurt am Main, Insel, 1993, S. 137.

48 Christoph Vögele (Hg.), Monica Studer, Christophe van Berg, Kunstmuseum Solothun, Zürich edition fink, 2005. 


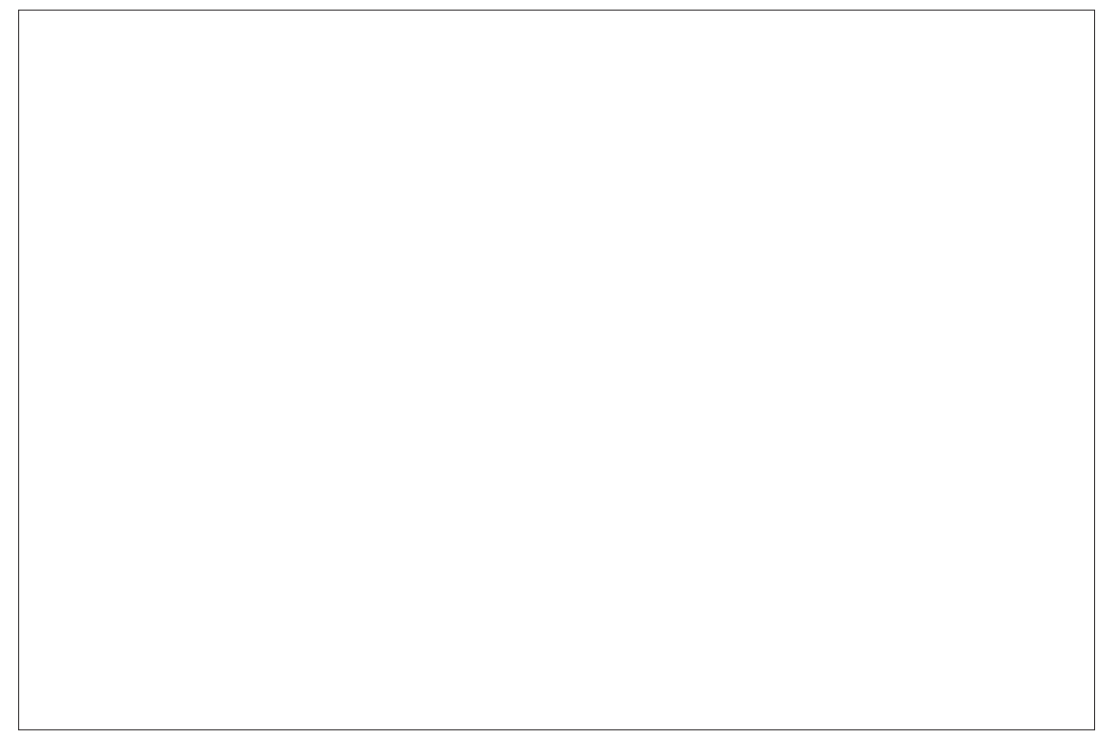

Monica Studer \& Christoph van den Berg, Loch (2009).

Beyond the picturesque exhibition at S.M.A.K. (Stedelijk Museum voor Actuele Kunst),

Gent, Belgium. Curators: Steven Jacobs \& Frank Maes.

Andacht geworden ist. Auf die durch die Rückenfigur angedeutete Wechselbeziehung von Ich und gotterfüllter Natur hat Walter Benjamin im romantischen Denken hingewiesen: „Im Medium der Reflexion gehen das Ding und das erkennende Wesen ineinander über, wobei zwischen Wahrnehmung und Erkenntnis nicht mehr unterschieden wird “ ${ }^{(49)}$.

Bei Monica Studer und Christophe van den Berg findet ein Transfer der Szenerie von Rügen an der Ostsee in die Alpen statt. „Die Großbilder sind keine herkömmlichen Ölbilder, sondern bestehen aus jeweils vier aneinander gefügten Inkjet-Prints - Tintenstrahl-Ausdrucken. Sie vollziehen einen weitgespannten kunst- und medienhistorischen Brückenschlag “ ${ }^{(50)}$. Sie versuchen, das Alpenmotiv über die Computerkunst zu erschließen, und gehen der Frage nach, wie persönliche Erinnerung und mediale Bilder bei der Wahrnehmung einer Landschaft zusammenwirken.

„Die Bandbreite, die dem Pluralismus des heutigen Kunstschaffens entspricht, reicht vom ironisch-distanzierten Umgang mit einem zum Klischee verkommenen Bild von den Alpen über rein formale Auseinandersetzungen bis hin zu den seriösen Versuchen, ihr Bild des Berges für eine neue, unverschlissene Erhabenheit aufrecht zu erhalten "(51). So ist das Alpenglühen wieder, wenn auch meist in ironischer und verfremdender Darstellungsweise, ein Motiv in zeitgenössischen Arbeiten. Beuys spricht von der mineralischen Kraft der Berge. Er meint, unter tiefenpsychologischem

49 Walter Benjamin, Der Begriff der Kunstkritik in der deutschen Romantik (1920), Frankfurt am Main, Suhrkamp, 1973, S. 52.

$50 \quad$ NZZ, 14.1.2006.

51 Stephan Kunz, Beat Wismer, Wolfgang Denk (Hg.), Die Schwerkraft der Berge 1774-1997, Katalog, Stroemfeld, Roter Stern, 1997, S. 11. 
Aspekt bedeute der Berg einen hohen Bewusstseinsgrad. Der Berg wird in Literatur und Malerei auch wieder als Ort der Sinn- und Wahrheitssuche gestaltet.

Alpen und innere Welten, Inner Light, den Erkenntisweg Berg, diese Vorstellungen zeigen Bill Violas ${ }^{(52)}$ Videokunst. Der führende Vertreter der Videokunst hat in der Ausstellung Sur les traces du sacré (Auf den Spuren des Heiligen) ${ }^{(53)}$ unter dem Titel Room for St. John of the Cross ${ }^{(54)}$ eine Inszenierung der Alpen kreiert, die an die mystische Tradition anknüpft. Der Zuschauer tritt in einen großen dunklen Raum und wird vom Tosen des Eissturms überrascht, der die monumentalen schwarz-weißen Berggipfel auf einer Riesenleinwand in heftige Bewegung versetzt. Dieser Aggression unvermutet ausgesetzt, entdeckt er mitten in dem dunklen Raum ein mildes Licht, eine kleine Hütte, in deren Innern er durch ein Fensterchen einen Tisch mit einem Krug Wasser und einem kleinen Bildschirm sieht, auf dem eine idyllische, friedliche Alpenlandschaft abgebildet ist. Eine sanfte Stimme rezitiert spanische Verse. Der Kontrast der sinnlichen Erfahrungsräume bewirkt ein Wechselbad der Gefühle; da die Emotion Bestandteil der ästhetischen Erfahrung ist, sollen so auf interaktive Weise die Aisthesis und Poiesis den Erkenntnisprozess und die Katharsis einleiten.

Auf der Suche nach einer neuen Ästhetik schreibt die junge Künstlergeneration den Alpenmythos weiter. Doch ihre Kreationen vermögen es nicht, die Ausdruckskraft des alpinen Zeichensystemes zu schmälern, das noch immer funktioniert. Zur Imagologie des schweizerischen Selbstverständnisses gehören unabdingbar die Alpen.

Die Untersuchung hat gezeigt, wie nationale Identitätsbilder von Geschichte, Politik und Wirtschaft bedingt sind. An Beispielen aus der bildenden Kunst konnte man sehen, wie Wilhelm Tell, einer der Helden der Geschichte, diese „Unsicherheiten, Banalitäten, Missverständnisse und Zufälle“ - um mit Aleida Assmann zu sprechen dekonstruiert wurde ${ }^{(55)}$. War er anfänglich eine nationale Integrationsfigur, so wurde seine Armbrust als Gütesiegel der Schweizer Industrie vermarktet und letztendlich mit Bezug auf Terrorismus und Kindesmissbrauch pervertiert.

Was das nationale Vorstellungsbild der Alpen angeht, so muss man differenzieren, denn es ist komplexer. Es bildet sozusagen das Urgestein schweizerischen Selbstverständnisses, war in der Malerei im 19. und bis in die Mitte des 20. Jahrhunderts stark national gewichtet, behält aber heute diese Rolle nur noch in geringerem Maße. Es konnte sinnfällig gemacht werden, wie die Alpen sich zum nationalen Symbol wandelten, vor allem seit der Entstehung des helvetischen Einheitsstaates als integrativer nationaler Mythos eine bedeutende Rolle spielten; ihre Idealisierung und Sakralisierung dienten dem nationalen Zusammenhalt. Das äußerte sich in der heroischen Alpenmalerei des 19. Jahrhunderts. Die vor dem Zweiten Weltkrieg einsetzende „Geistige Landesverteidigung“ (GLV) benutzte die Kunst zur Schaffung einer nationalen Identität.

52 Bill Viola (1951 New York), amerikanischer Videokünstler.

53 Ausstellung Sur les traces du sacré (Auf den Spuren des Heiligen) im Centre Pompidou in Paris, 7. Mai-11. August 2008. Dort war auch Ferdinand Hodlers Blick ins Unendliche (1905) zu sehen.

54 Bill Viola 1983, Video-Installation, 426,7 cm x 731,5cm x 914,4 cm, Coll. MoCA, Los Angeles.

55 Aleida Assmann, „Die (De-)Konstruktion nationaler Mythen und die Rolle der Literatur“, in: Corina Caduff, Reto Sorg (Hg.), Nationale Literaturen heute - ein Fantom? Die Imagination und Tradition des Schweizerischen als Problem, München, Fink, 2004, S. 75-83, hier S. 80. 
„Diese Symbiose zwischen Kunst und Nation löste sich in den 50er Jahren auf, weil sich die Künstler immer mehr an internationalen Strömungen ausrichteten und den Landesgrenzen keine Bedeutung mehr beimaßen“( ${ }^{(56)}$. Hatten sich noch die von Peter von Matt als Mythenbekämpfer, Mythenbeseitiger und Bilderzertrümmerer bezeichneten Autoren Max Frisch und Friedrich Dürrenmatt ${ }^{(57)}$ mit den Vorstellungsbildern der Schweiz auseinander gesetzt, so forderte die Generation der 80er ein neue Offenheit. Zwar wurden die Alpen stets durch Ideologien und Wirtschaftsinteressen vereinnahmt und dienen den Zwecken nationaler Selbstinszenierung. Doch Malerei und bildende Kunst schrieben den Mythos neu, auf der Suche nach einer innovativen alpinen Ästhetik, sei es über die Photographie, die Video- oder die Computerkunst.

Es gibt eine reflexive Arbeit am Mythos, ein „Um- und Fortschreiben des Mythos Schweiz"(58), was sich auch in der bildenden Kunst zeigt. Die Alpen lassen sich nicht von politischen, ökonomischen Diskursen vereinnahmen, doch allein die Permanenz des Dialogs Alpen und Malerei dokumentiert das Alpine als ein atemporales Faszinosum.

\section{Résumé}

Le discours à motivation patriotique sur les Alpes était une caractéristique constitutive de la peinture suisse; les représentations des Alpes comme phénomène de l'histoire culturelle permettent d'établir des liens avec les structures identitaires nationales et avec la mémoire collective. Ainsi, les scènes de montagnes hérö̈ques du XIX ${ }^{e}$ siècle (Alexandre Calame) ainsi que l'iconographie de l'homo alpinus helveticus (Le Tell de Richard Kissling ou de Ferdinand Hodler) remplissent la fonction d'icône nationale.

Dès le tournant $d u X^{e}$ siècle, des discours subversifs sur les Alpes (Karl Walser) provoquent l'érosion de l'image surdimensionnée que la Suisse se fait d'elle-même; depuis 1945, des discours picturaux opposés aplanissent le mythe des Alpes, déconstruisent - au sens de Roland Barthes - l'idéologie alpine (Gerhard Richter, Adam Tellmeister e.a.). Le slogan utopique des années 80 "Enlevez les Alpes, horizon dégagé sur la Méditerranée " fait place à l'ancrage du Discours à l'étroit de Paul Nizon dans l'emblématique alpine. La fuite hors de l'étroitesse et de l'enfermement que produit la conscience du statut particulier du pays alpin suisse trouve son expression dans le domaine des arts plastiques à travers le pluralisme du discours sur les Alpes. À celui-ci correspond la variété des techniques artistiques que mettent en cuvre des artistes de nationalités diverses (Monica Studer et Christoph van den Berg, Bill Viola e.a.).

\section{Zusammenfassung}

Der patriotisch motivierte Alpendiskurs war ein konstitutives Merkmal der Schweizer Malerei; die Alpendarstellungen als kulturgeschichtliches Phänomen ermöglichen es, Bezüge zu nationalen Identitätsstrukturen und zum kulturellen Gedächtnis herzustellen.

56 www.schweizimspiegelderwelt.ch/die-nation-im-spiegel-der-kunst.html

57 Peter von Matт, Die tintenblauen Eidgenossen. Über die literarische und politische Schweiz, München, Carl Hanser Verlag, 2001, S. 225-240, hier S. 226.

58 Jürgen Barkhoff, Valerie HefFernan (Hg.), Schweiz schreiben: Zu Konstruktion und Dekonstruktion des Mythos Schweiz in der Gegenwartsliteratur, Tübingen, Niemeyer, 2010, S. 7. 
So fungieren sie in den heroischen Gebirgsszenen des 19. Jahrhunderts (Alexandre Calame), wie die damalige Ikonographie des homo alpinus helveticus (Tell-Figur Richard Kisslings oder Ferdinand Hodlers) als nationales Motiv.

Bereits um die Jahrhundertwende erodieren implizit subversive Alpendiskurse das überdimensionale Selbstbild der Schweiz (Karl Walser) und seit 1945 planieren Gegendiskurse in der Malerei den Alpen-Mythos, dekonstruieren - im Sinne Roland Barthes - die Alpenideologie (Gerhard Richter, Adam Tellmeister u.a.). Der utopische Slogan der 80er Jahre „Weg mit den Alpen - freie Sicht aufs Mittelmeer!“ verortet Paul Nizons Diskurs in der Enge in der Alpenemblematik. Die Flucht aus der Enge des Sonderstatusbewusstseins des Schweizeralpenlandes drückt sich im Bereich der Bildenden Künste durch den Pluralismus der Alpendiskurse aus. Diesem entspricht die Vielfalt neuer künstlerischer Techniken die Kunstschaffende verschiedenster Nationalitäten (Monica Studer und Christoph van den Berg, Bill Viola u.a.) ins Werk setzen.

\begin{abstract}
Patriotically-motivated discourse on the Alps was a fundamental characteristic of Swiss painting; the representations of the Alps as a phenomenon of cultural history permitted the establishment of links with national identity structures and with collective memory. Thus 19th Century scenes of grandiose mountains (Alexandre Calam) and the iconography of homo alpinus helveticus (the Tell of Richard Kissling or of Ferdinand Hodler) took on a national iconic function.

At the turn of the 20th Century, subversive discourses on the Alps were already beginning to erode the oversized Swiss self-image; since 1945, opposing pictorial discourses have flattened out the Alpine myth and deconstructed - in the sense given to this term by Roland Barthes - Alpine ideology (Gerhard Richter, Adam Tellmeister, amongst others). The utopian slogan of the 80s, "Remove the Alps and open the horizon to the Mediterranean", has taken the place of Paul Nizon's Discourse of the Narrow in the Alpine emblematic. The escape beyond narrowness and enclosure in the perception of the Swiss Alpine landscape's special status finds its expression in the field of the visual arts through a pluralistic discourse on the Alps and a corresponding variety of artistic techniques exploited by artists of different nationalities (Monica Studer and Christoph van den Berg, Bill Viola, amongst others).
\end{abstract}

